\title{
Arzneimittelinteraktionen, die man kennen muss!
}

N. Voigt, K. Ort, S. Sossalla

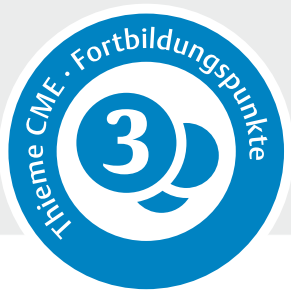

\section{Arzneimittelinteraktionen (AMI) sind ein häufig unterschätztes Problem der} modernen Medizin. Die Zahl älterer, multimorbider Patienten, die mehrere Wirkstoffe gleichzeitig einnehmen, nimmt stetig zu - der damit verbundene exponentielle Anstieg möglicher AMI ist fast unüberschaubar. Diese Übersicht stellt häufige, relevante AMI anhand von Beispielen dar und zeigt Strategien auf, wie sie in der Praxis frühzeitig erkannt und vermieden werden können.

$\begin{array}{ll}\text { ABKÜRZUNGEN } \\ \text { ACE } & \text { Angiotensin-converting Enzyme } \\ \text { AM } & \text { Arzneimittel } \\ \text { AMI } & \text { Arzneimittelinteraktionen } \\ \text { ASS } & \text { Azetylsalizylsäure } \\ \text { COX-1 } & \text { Cyclooxygenase-1 } \\ \text { CYP } & \text { Cytochrom-P450-System } \\ \text { LQT } & \text { Long QT } \\ \text { NOAK } & \text { nicht-Vitamin-K-abhängige orale Anti- } \\ & \text { koagulanzien } \\ \text { NSAR } & \text { nichtsteroidale Antirheumatika } \\ \text { OATP } & \text { Organic Anion Transporting Protein } \\ \text { PDE } & \text { Phosphodiesterase } \\ \text { P-gP } & \text { P-Glykoprotein } \\ \text { PPI } & \text { Protonenpumpeninhibitoren } \\ \text { SERT } & \text { Serotonintransporter } \\ \text { SSRI } & \text { selektive Serotonin-Rückaufnahme- } \\ & \text { Inhibitoren } \\ \text { TdP } & \text { Torsade-de-Pointes-Tachykardie } \\ & \end{array}$

\section{Einleitung}

Es wird angenommen, dass 1 - $5 \%$ aller Krankenhauseinweisungen auf Arzneimittelinteraktionen (AMI) zurückzuführen sind oder zumindest mit diesen in Zusammenhang stehen [1]. Hochgerechnet auf die Anzahl an jährlichen Krankenhauseinweisungen (20 Mio. Patienten) sind in Deutschland daher 200000 bis 1 Mio. Patienten pro Jahr betroffen. In unserer alternden Gesellschaft werden immer mehr Arzneimittel (AM) in Kombination eingenommen (Polypharmazie). Vor diesem Hintergrund fällt es in der täglichen Praxis zunehmend schwerer, alle möglichen AMI zu kennen und zu vermeiden [2].
Für den vorliegenden Beitrag haben wir daher besonders häufige und schwerwiegende AMI systematisch zusammengefasst [1]. Wir haben AMI ergänzt, die uns aus eigener Erfahrung besonders erwähnenswert scheinen oder die bestimmte Interaktionsprinzipien einprägsam darstellen.

\section{Pharmakodynamische Interaktionen}

\section{Synergistische pharmakodynamische Interaktionen}

Bei Kombination von Pharmaka mit gleichem Wirkmechanismus kann es zur synergistischen Wirkverstärkung, aber auch zur wechselseitigen Hemmung der AMWirkung kommen. Pharmakodynamische AMI können die Auftretenswahrscheinlichkeit sonst kaum beobachteter unerwünschter Arzneimittelwirkungen derart erhöhen, dass diese klinische Relevanz erhalten. Pharmakodynamische AMI sind ferner nicht mit Veränderungen der Wirkstoffkonzentration verbunden. Auch sind meist alle Vertreter einer Substanzklasse betroffen (Klassenphänomene), da die Interaktion auf den Wirkmechanismus der Substanz zurückzuführen ist (

\section{NO-Donatoren vs. Phosphodiesterasehemmer}

Ein sehr prominentes Beispiel für eine synergistische pharmakodynamische Interaktion ist die additive vasodilatatorische Wirkung von

- bei erektiler Dysfunktion zum Einsatz kommenden Phosphodiesterase (PDE)-5-Hemmern

(z. B. Sildenafil) und

- organischen Nitraten sowie Molsidomin (NO-Donatoren).

Beide Medikamentengruppen greifen in den Metabolismus des zyklischen Guanosinmonophosphats (cGMP), einem wichtigen Mediator der Relaxation glatter Gefäßmuskeln, ein. 
- Tab. 1 Systematik und Beispiele pharmakodynamischer Interaktionen (Wirkungsänderung ohne Veränderung der Konzentration, Klassenphänomen).

\begin{tabular}{|l|l|}
\hline synergistisch & antagonistisch \\
\hline & \\
\hline - PDE-5-Hemmer+Nitrate & - Azetylsalizylsäure+ \\
\hline - Betarezeptorblocker+ & Ibuprofen \\
\hline $\begin{array}{l}\text { Kalziumantagonisten } \\
\text { (Verapamil, Diltiazem) }\end{array}$ & - Antidiabetika+Cluko- \\
\hline - SSRI+Antikoagulanzien & kortikoide \\
\hline
\end{tabular}

PDE: Phosphodiesterase, SSRI: selektive Serotonin-Rückaufnahme-Inhibitoren

\section{Cave \\ Bei gleichzeitiger Gabe von NO-Donatoren und PDE- 5-Hemmern wird die gefäßdilatierende Wirkung beider Wirkstoffgruppen potenziert und es kann zu lebensbedrohlichen Blutdruckabfällen kommen.}

So führte in einer Studie die Gabe von Tadalafil ( $4 \mathrm{mg}$ ) bei $26 \%$ der mit Nitroglycerin $(0,4 \mathrm{mg})$ behandelten Patienten zu signifikanten Blutdruckabfällen, d. h. systolischer Blutdruck $<85 \mathrm{mmHg}$. Hingegen kommt es bei Kombination von PDE-5-Inhibitoren mit anderen antihypertensiv wirkenden Pharmaka lediglich zu einer moderaten additiven Blutdrucksenkung von ca. $4 \mathrm{mmHg}$ systolisch und $2 \mathrm{mmHg}$ diastolisch. Dazu gehören Kalziumantagonisten, Betarezeptorblocker, ACE-Hemmer und Diuretika [3]. Vorsicht ist ebenfalls geboten bei Kombination von PDE-5-Hemmern mit Alphablockern, die auch zu einem signifikanten Blutdruckabfall führen können. Dabei fallen die hypotensiven Effekte bei den sog. „uro-selektiven“ Alphablockern (z. B. Tamsulosin) geringer aus, weshalb diese zu bevorzugen sind [4].

\section{Betarezeptorblocker und Kalziumantagonisten vom Verapamil- und Diltiazem-Typ}

Eine weitere klassische synergistische pharmakodynamische Interaktion ist die Wechselwirkung

- von Kalziumantagonisten vom Verapamil- und Diltiazemtyp (Phenylalkylamine und Benzothiazine)

- mit anderen, die AV-Überleitung verzögernden

Wirkstoffen, insbesondere Betarezeptorblockern.

Es ist allgemein bekannt, dass diese Kombination zu einer erheblichen AV-Überleitungsverzögerung bis hin zum AV-Block III führen kann [2]. Wir möchten darauf hinweisen, dass bei der Beurteilung möglicher AMI auch nichtorale Applikationsformen von Betarezeptorblockern, wie Augentropfen, in Betracht gezogen werden müssen (s. „Fallbeispiel - AV-Überleitungsverzögerung“ [5]).
FALLBEISPIEL

\section{AV-Überleitungsverzögerung}

Bei einem 64-jährigen Patienten fällt bei einer Routineuntersuchung eine Bradykardie von 36 Schlägen pro Minute auf. Zur Prophylaxe von Glaukom-Anfällen erhält er 2-mal täglich Timolol Augentropfen (0,5\%). Außerdem nimmt er seit 12 Monaten Verapamil aufgrund von Angina bei chronischer koronarer Herzerkrankung ein. Timolol, ein Betarezeptorblocker, der zur Prophylaxe von Glaukom-Anfällen als Augentropfen eingesetzt wird, weist aufgrund der Umgehung des Pfortader-Kreislaufs eine sehr hohe Bioverfügbarkeit (80\%) auf. Bei gleichzeitiger Applikation von Verapamil mit dem Betarezeptorblocker kann es daher zu signifikanten Bradykardien durch Addierung der negativ dromotropen Effekte beider Substanzen kommen. Nach Umstellung der Medikation von Verapamil auf Nifedipin normalisiert sich die Herzfrequenz auf 78 Schläge pro Minute [5] (heute sind anstelle von Nifedipin langsamer anflutende Kalziumantagonisten wie Amlodipin oder Lercanidipin eher üblich).

\section{SSRI und Antikoagulanzien}

In den letzten Jahren haben sich Hinweise auf ein erhöhtes Blutungsrisiko durch die Einnahme von selektiven Serotonin-Rückaufnahme-Inhibitoren (SSRI, z.B. Citalopram, Fluoxetin, Paroxetin) verdichtet.

Ursache ist offenbar eine Interaktion mit dem Serotonintransporter (SERT) in Thrombozyten ( $\mathbf{A b b . 1 )}$ ). Serotonin stellt einen wichtigen Mediator der Thrombozytenaggregation dar. SSRI hemmen die Aufnahme von Serotonin nicht nur an serotoninergen Neuronen, sondern offenbar auch an Thrombozyten [6]. Es kommt zu einer gestörten Thrombozytenaggregation. Erste Anhaltspunkte lieferte 2012 eine kanadische Metaanalyse, in der 16 Studien mit Daten von mehr als einer halben Million Patienten analysiert wurden [7]. Eine SSRITherapie erhöht demzufolge die Gefahr, eine intrakranielle Hämorrhagie zu erleiden, um rund $50 \%$. Nahmen die Patienten zusätzlich Antikoagulanzien ein, stieg das Hirnblutungsrisiko im Vergleich zu einer Antikoagulanzien-Monotherapie um weitere $56 \%$. Dies ist vergleichbar mit der Erhöhung des Hirnblutungsrisikos durch Wechselwirkung von Azetylsalizylsäure (ASS), Clopidogrel oder anderen Thrombozyten-wirksamen Arzneistoffen mit oralen Antikoagulanzien.

Ein erhöhtes Risiko für gastrointestinale Blutungen offenbarte eine Metaanalyse von 4 Beobachtungsstudien von Patienten, die mit SSRI und nichtsteroidalen 


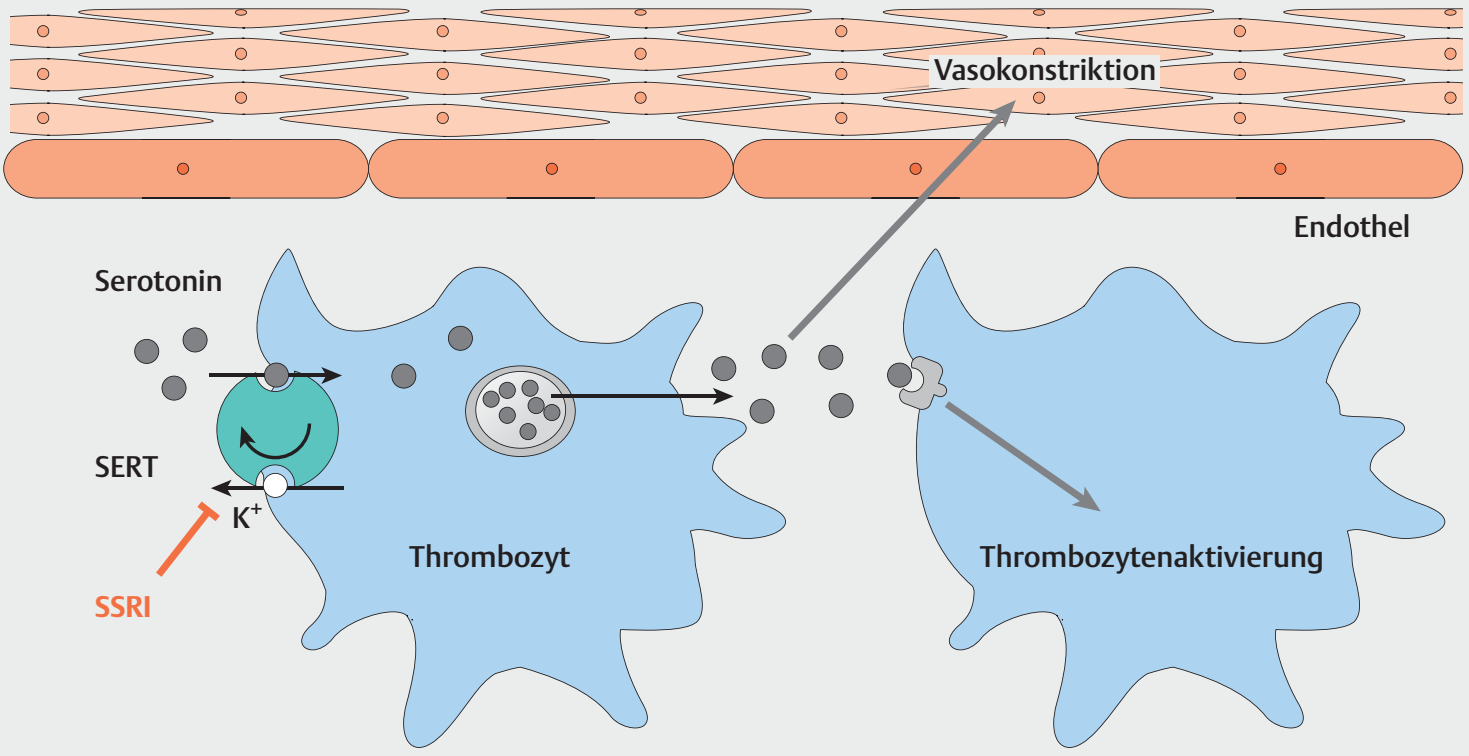

- Abb. 1 Selektive Serotonin-Rückaufnahme-Inhibitoren (SSRI) interagieren mit der Serotoninaufnahme in Thrombozyten. Die Freisetzung von Serotonin aus Thrombozyten spielt eine wichtige Rolle während der Thrombozytenaggregation. Das aus Thrombozyten freigesetzte Serotonin löst an der glatten Gefäßmuskulatur eine Vasokonstriktion aus und aktiviert weitere Thrombozyten. Durch Hemmung der Serotoninaufnahme in Thrombozyten (Serotonintransporter, SERT) können SSRI das Blutungsrisiko bei gleichzeitiger Einnahme von Antikoagulanzien überproportional erhöhen.

Antirheumatika (NSAR) behandelt wurden [8]. Unter SSRI-Therapie war das Risiko gastrointestinaler Blutungen verdoppelt, unter alleiniger NSAR-Therapie 3-fach und unter der Kombination aus SSRI und NSAR 6-fach erhöht.

\section{Merke \\ Patienten mit Hirnblutungen, gastrointestinalen Blutungen und Magengeschwüren in der Vergangen- heit sollten - wenn möglich - mit Antidepressiva behandelt werden, die geringere Affinität zum SERT aufweisen als SSRI.}

\section{QT-Zeit-verlängernde Pharmaka}

Die Verlängerung der QT-Zeit im EKG gilt als Indikator für ein erhöhtes Risiko für Torsade-de-Pointes-Tachykardien (TdP) [9]. Die einzige Substanzklasse QT-Zeitverlängernder Pharmaka, für die ein vermehrtes Auftreten von TdP-Arrhythmien eindeutig nachgewiesen ist, sind Antiarrhythmika der Klassen IA (z. B. Chinidin) und Klasse III (z. B. Sotalol) [10]. Bei anderen Pharmaka, wie Makrolidantibiotika und Antipsychotika, gelten die Verlängerung der QTZeit (Long-QT, LQT) und Blockade von IKr-Kanälen als Surrogat-Marker für ein erhöhtes Risiko für TdP-Arrhythmien.

QT-Zeit-verlängernde Pharmaka werden nach der AZCERT-Klassifikation (Arizona Center for Education and Research on Therapeutics, s. „Praxis - Online Tools“) in 3 Kategorien unterteilt:
- Zum einen in AM mit „bekanntem“ Risiko, wobei die QT-Zeit-Verlängerung regelmäßig dosisabhängig TdP-Arrhythmien auslöst (s. „Info - LQT-Pharmaka: 5-A-Regel“).

- Die zweite Gruppe umfasst AM mit „möglichem“ Risiko für TdP-Arrhythmien. Diese können zwar regelmäßig eine QT-Zeit-Verlängerung auslösen, lösen aber nicht nachweislich oder nur sehr selten TdP-Arrhythmien aus (z. B. Clozapin, Tramadol).

- Die dritte Gruppe fasst AM zusammen, bei denen das Risiko einer QT-Zeit-Verlängerung und Auslösung von TdP-Arrhythmien vom Vorhandensein zusätzlicher Ko-Faktoren (z. B. Hypokaliämie oder genetische Prädisposition) abhängt („,bedingtes“ Risiko, z. B. Diphenhydramin, Omeprazol, Loperamid).

Insbesondere bei älteren Patienten ist mitunter die gleichzeitige Einnahme mehrerer die QT-Zeit verlängernde AM unumgänglich. In gängigen Arzneimittelinteraktionsdatenbanken (s. „Praxis - Online Tools“) wird bei Verordnung von QT-Zeit-verlängernden AM vor synergistischen pharmakodynamischen Effekten gewarnt. Diese Warnungen müssen differenziert betrachtet werden: Die Annahme, dass sich die QT-Zeit umso stärker verlängert, je mehr QT-Zeitverlängernde AM eingenommen werden und das Risiko für TdPArrhythmien entsprechend ansteigt, ist nicht ausreichend belegt. Lediglich für die Kombination zweier AM mit „bekanntem Risiko“ für TdP-Arrhythmien konnte eine additive Wirkung auf die QT-Zeit-Verlängerung 
nachgewiesen werden. Dass dies nicht zwangsläufig mit einem höheren Risiko für TdP-Arrhythmien einhergehen muss, zeigt eine neuere Studie: Hier erhöhte die Gabe eines weiteren QT-Zeit-verlängernden AM das Risiko für das Auftreten von TdP-Arrhythmien nicht signifikant [11].

Vor diesem Hintergrund sollte also die Kombination von diesen AM nicht per se ausgeschlossen werden. Vielmehr sollte das individuelle Nutzen-Risiko-Verhältnis abgewogen werden und ein entsprechendes QTMonitoring erfolgen. Hierbei sind zusätzliche Faktoren zu berücksichtigen wie höheres Lebensalter, weibliches Geschlecht, genetische Prädisposition, strukturelle Herzerkrankungen und Elektrolytverschiebungen (insbesondere Hypokaliämie, Cave: Diuretika) [12].

\section{INFO}

LQT-Pharmaka: 5-A-Regel

Die 5 „A“-Gruppen der Medikamente mit „,bekanntem Risiko“ für TdP-Arrhythmien sind:

- Antiarrhythmika (Amiodaron, Chinidin, Dronedaron, Flecainid, Sotalol)

- Antidepressiva (Citalopram, Escitalopram)

- Antipsychotika (Chlorpromazin, Droperidol, Haloperidol, Levomepromazin, Sulpirid, Thioridazin)

- Antibiotika (Fluorchinolone: Ciprofloxacin, Levofloxacin, Moxifloxacin; Makrolide: Azithromycin, Clarithromycin, Erythromycin, Roxithromycin)

- Andere (Chloroquin, Domperidon, Donepezil, Fluconazol, Methadon, Propofol)

\section{Antagonistische pharmakodynamische Interaktionen}

\section{Azetylsalizylsäure und NSAR}

ASS ist der wichtigste Thrombozytenaggregationshemmer und zählt zu den am meisten verwendeten AM. Die thrombozytenaggregationshemmende Wirkung ist im Wesentlichen auf eine Acetylierung und damit irreversible Hemmung der thrombozytären Cyclooxygenase-1 (COX-1) zurückzuführen. Da Thrombozyten keinen Zellkern besitzen, um dieses für die Thrombozytenaggregation wichtige Enzym nachzusynthetisieren, bleibt die aggregationshemmende Wirkung während der gesamten Thrombozyten-Lebensdauer erhalten. So kann trotz der geringen Halbwertszeit von ASS (ca. $15 \mathrm{~min}$ ) eine langanhaltende thrombozytenaggregationshemmende Wirkung erzielt werden (Wirkverlust nach ca. 5 Tagen).

NSAR wie Ibuprofen binden reversibel an die COX-1 der Thrombozyten und verhindern so die Acetylierung und irreversible Hemmung der thrombozytären COX-1 durch ASS. Ausmaß und Dauer der Hemmung der Thrombozytenaggregation werden so eingeschränkt und das kardiale Risiko für Patienten mit koronarer Herzkrankheit steigt [2]. In-vitro-Experimente konnten zeigen, dass nicht alle NSAR von dieser Interaktion betroffen sind: So scheinen Paracetamol oder Diclofenac die thrombozytenaggregationshemmende Wirkung von ASS nicht zu beeinflussen [13].

Merke

Einmalige Einnahme von NSAR unter ASS-Therapie scheint unbedenklich. Bei häufiger Einnahme sollten NSAR mindestens $8 \mathrm{~h}$ vor und frühestens $30 \mathrm{~min}$ nach ASS eingenommen werden. Paracetamol scheint die thrombozytenaggregationshemmende Wirkung von ASS nicht zu beeinflussen.

\section{Pharmakokinetische Interaktionen}

Unter pharmakokinetischen AMI werden im Folgenden Interaktionen zusammengefasst, die die Resorption, Verteilung, Metabolisierung und Elimination beeinflussen und somit die effektive Konzentration am Wirkort verändern.

\section{Interaktionen bei der Resorption}

P-Glykoprotein-vermittelte Interaktionen

P-Glykoprotein (P-gp) wird an vielen Gewebebarrieren exprimiert, wie beispielsweise der Blut-Hirn-Schranke sowie dem Nieren- und Darmepithel. P-gp transportiert lipophile Pharmaka wie Digoxin aus dem Zytosol über die apikalen Zellmembranen nach außen. Beispielsweise kann eine Hemmung von P-gp durch Verapamil zu einer verstärkten oralen Bioverfügbarkeit von Digoxin beitragen. Bei Dabigatranetexilat, der inaktiven Vorstufe des nicht-Vitamin-K-abhängigen (früher: „neuen“) oralen Antikoagulans (NOAK) Dabigatran, ist P-gp verantwortlich für die relativ geringe Bioverfügbarkeit (ca. 7\%). Daher ist bei Kombination mit Inhibitoren (z. B. Verapamil) bzw. Induktoren (z. B. Rifampicin) des P-gp mit erhöhten bzw. verminderten Dabigatran-Plasmaspiegeln zu rechnen ( $\triangleright$ Abb.2).

\section{Merke}

In aktuellen Leitlinien wird eine Dosisreduktion des NOAK Dabigatran bei Kombination mit Verapamil, Amiodaron oder Chinidin empfohlen [14].

\section{Interaktionen bei der Verteilung}

AMI durch Konkurrenz verschiedener Pharmaka um die Plasmaeiweißbindung wurden lange überschätzt. Wenn überhaupt, spielt die gegenseitige Verdrängung von AM eine Rolle bei Pharmaka mit hoher Plasmaeiweißbindung, geringem Verteilungsvolumen und en- 


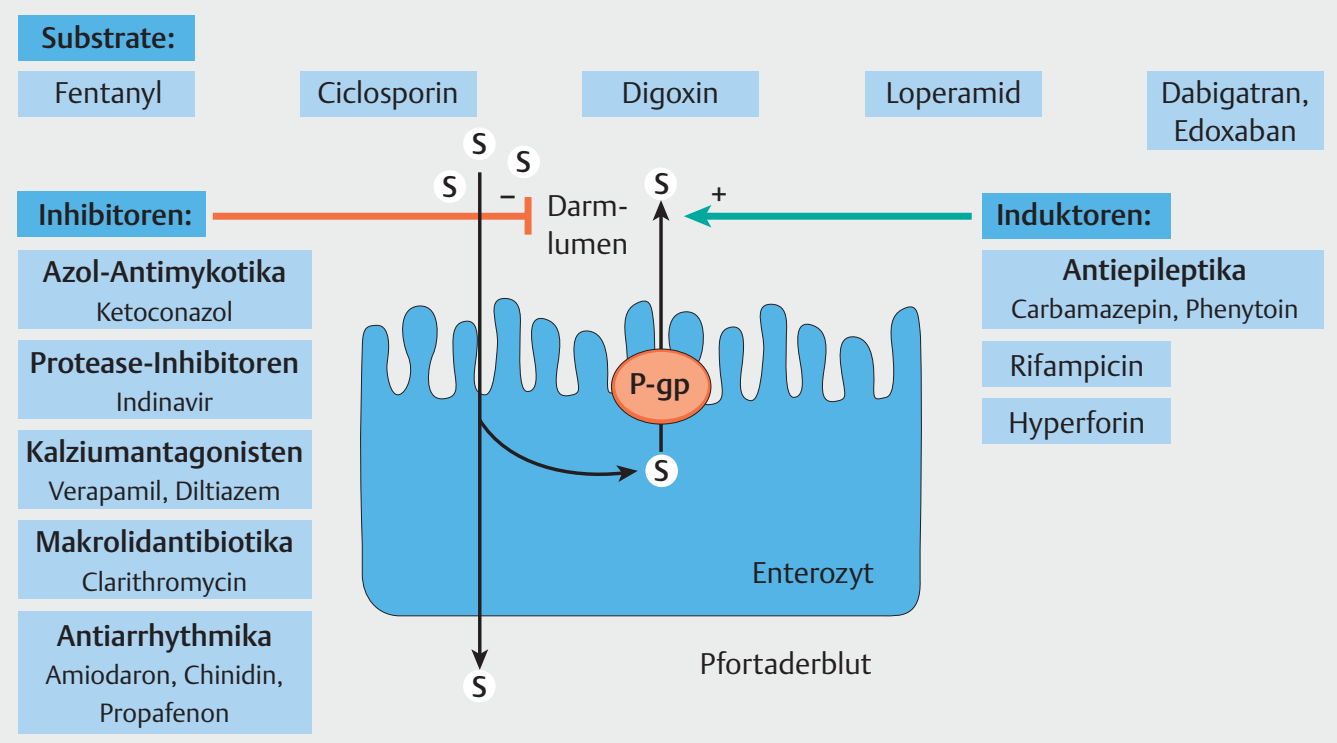

- Abb.2 Substrate, Inhibitoren und Induktoren des P-Glykoproteins (P-gP). S: Substrat, Hyperforin: Hauptwirkstoff in Johanniskrautextrakten.

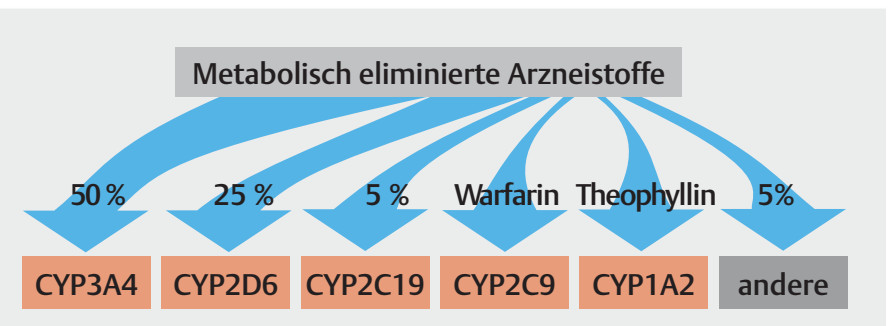

Abb. 3 Wichtige Iso-Enzyme des Cytochrom-P450-Systems. Die Darstellung zeigt, wie viel Prozent der metabolisch eliminierten Arzneistoffe über das jeweilige Iso-Enzym eliminiert werden.

gem therapeutischem Bereich. Hierzu zählen: Phenprocoumon, Sulfonylharnstoffe, Phenytoin, Diazepam und Digitoxin [15].

\section{Interaktionen bei der Metabolisierung und Ausscheidung}

\section{Organic Anion Transporting Protein: OATP1B1}

Eine wichtige Rolle bei der Metabolisierung und Ausscheidung von AM spielt die Aufnahme des AM in Leberund Nierenepithelzellen. Diese wird über organische Anionen-(OAT) und Kationentransporter (OCT) vermittelt. Neben körpereigenen Stoffen wie Gallensäuren, Schilddrüsenhormonen, Prostaglandinen und Katecholaminen werden über diese Transporter auch AM aufgenommen. So werden beispielsweise über OATP1B1 (Organic Anionic Transporting Polypeptide 1B1) bestimmte Statine, ACE-Hemmer und Angiotensinrezeptor-Antagonisten aus dem Pfortader-Kreislauf in die Hepatozyten aufgenommen. Bei Hemmung dieses ers- ten Schrittes in der hepatischen Elimination, z. B. durch Ciclosporin oder Fibrate, kommt es zu

- einer gestörten hepatischen Aufnahme,

- einer erhöhten Bioverfügbarkeit und folgend

- einem gesteigerten Risiko für unerwünschte Arzneimittelwirkungen (s. „Fallbeispiel - Cerivastatin“ [16]).

\section{FALLBEISPIEL \\ Cerivastatin}

Im August 2001 musste die Firma Bayer den Lipidsenker Cerivastatin (Lipobay ${ }^{\circledR}$ ) vom Markt nehmen, nachdem zahlreiche Todesfälle unter Cerivastatin bekannt geworden waren. Diese standen mit der gleichzeitigen Einnahme von Cerivastatin und Gemfibrozil als Lipidsenker in Zusammenhang. Als Ursache wurde eine OATP1B1-vermittelte Interaktion identifiziert. Gemfibrozil hemmt die Aufnahme und damit den Abbau von Cerivastatin in Hepatozyten. Die deutlich erhöhte Bioverfügbarkeit von Cerivastatin führte zu einem gesteigerten Risiko für oft tödlich verlaufende Rhabdomyolysen [16]

\section{Cytochrom-P450-Enzyme}

Viele AM werden über das Cytochrom-P450-System (CYP) metabolisiert. AM, aber auch Nahrungsmittel und andere Substanzen, können die Aktivität des Systems im Sinne einer Hemmung (Inhibitoren) oder Aktivitätssteigerung (Induktoren) modulieren. Hierdurch bedingt kommt es zu vielfältigen AMI. Die Komplexität 

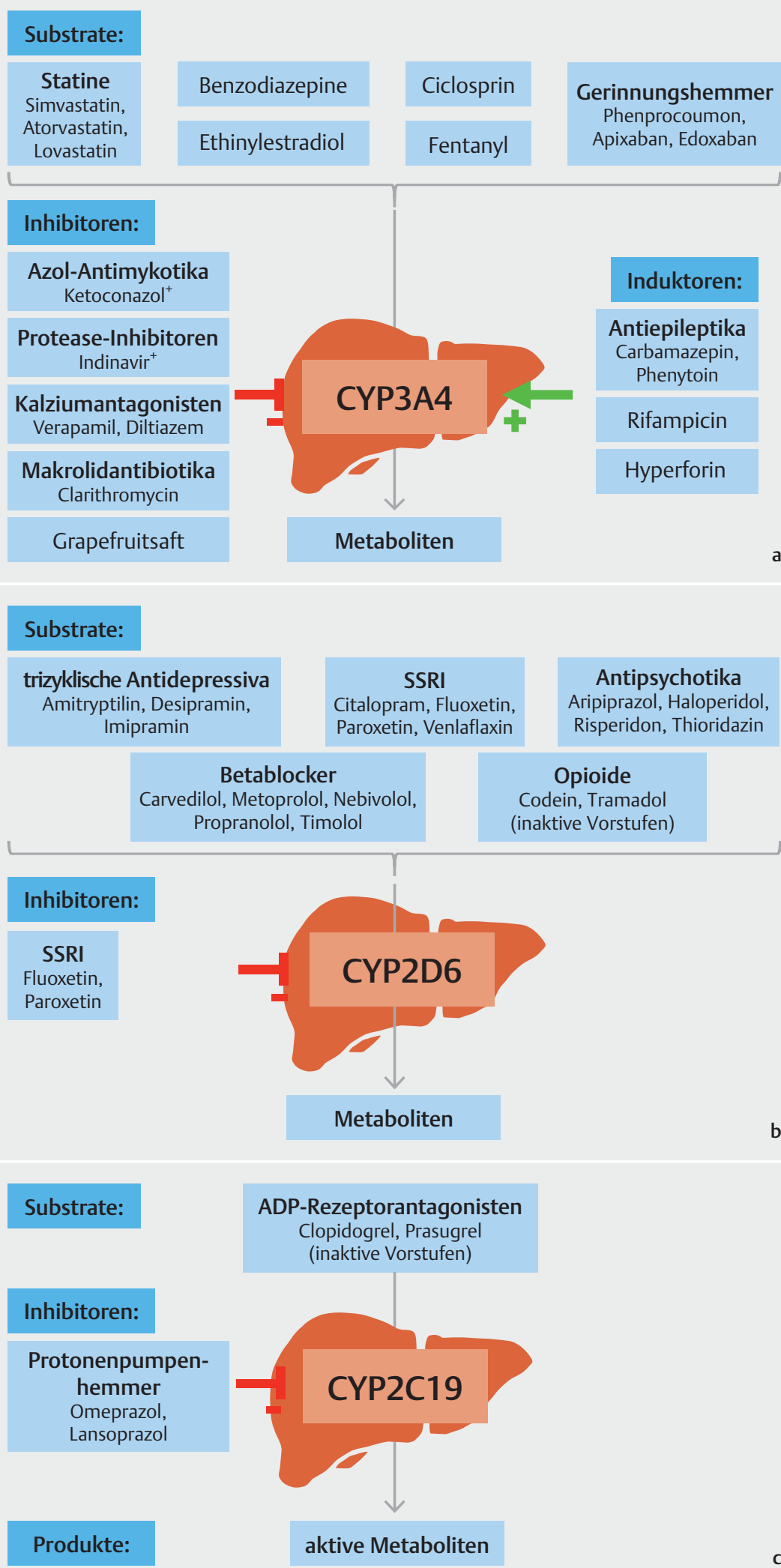

- Abb. 4 Substrate, Inhibitoren und Induktoren der 3 wichtigsten CYP-Iso-Enzyme. a CYP3A4. Ketoconazol und Indinavir (+) gelten als besonders starke Inhibitoren. Hyperforin: Hauptwirkstoff in Johanniskrautextrakten. b Substrate und Inhibitoren von CYP2D6. SSRI: selektive Serotonin-Rückaufnahme-Inhibitoren. c Substrate und Inhibitoren von CYP2C19. ADP: Adenosindiphosphat. 


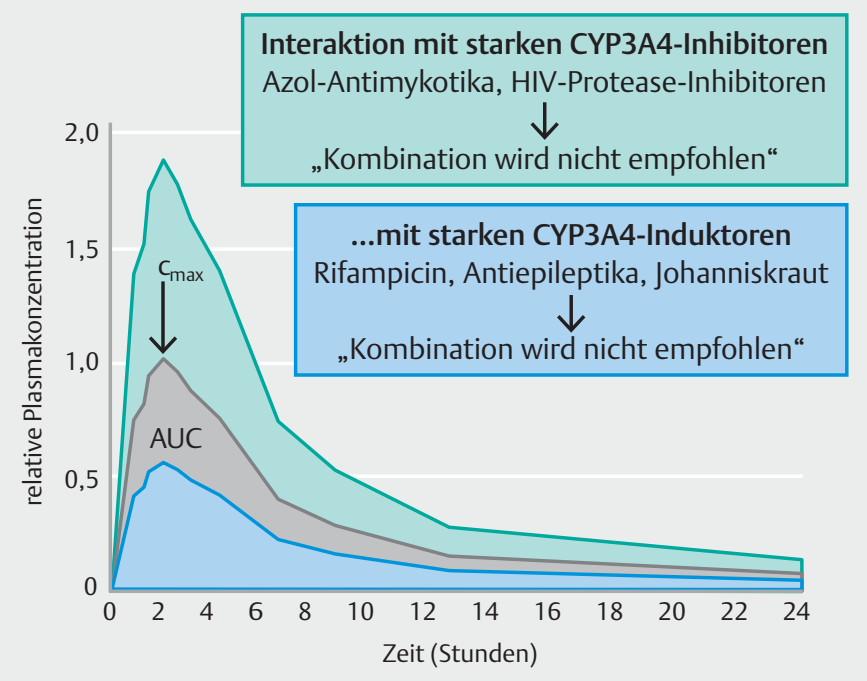

- Abb.5 Schematische Darstellung der pharmakokinetischen Interaktionen von nicht-Vitamin-K-abhängigen oralen Antikoagulanzien (NOAK) mit CYP3A4. Die Kombination von Rivaroxaban oder Apixaban mit starken CYP3A4-Inhibitoren oder -Induktoren wird nicht empfohlen. Wirkstoffe mit mäßiger Hemmwirkung (Diltiazem, Naproxen, Amiodaron, Verapamil) erhöhen die Plasmakonzentration der NOAK dagegen nur in geringem Maße. Hier ist ggf. lediglich eine Dosisreduktion erforderlich. Beim Metabolismus von Dabigatran und Edoxaban spielt CYP3A4 nur eine geringe Rolle, trotzdem sind Interaktionen über P-Glykoprotein zu beachten ( Abb.2). $C_{\max }$ : maximale Plasmakonzentration, AUC: Fläche unter der Kurve [14].

des Systems wird außerdem durch das Vorhandensein verschiedener CYP-Iso-Enzyme ( $\boldsymbol{A} \mathbf{A b} \mathbf{b}$. $\mathbf{3}$ ) erhöht, wobei jedes Iso-Enzym ein eigenes Spektrum an Substraten, Inhibitoren und Induktoren aufweist. Dies führt zu einer schier unüberschaubaren Fülle an Interaktionsmöglichkeiten, die in diversen Internetdatenbanken zusammengefasst und regelmäßig aktualisiert werden (s. „Praxis - Online Tools“). Folgende Überlegungen haben wir genutzt, um eine Auswahl an wichtigen AMI zu treffen und hier zusammenzufassen:

\footnotetext{
Merke

Die wichtigsten CYP-vermittelten AMI, die man kennen muss [17], betreffen:
}

- häufig verordnete Inhibitoren oder Induktoren und - Substrate mit geringer therapeutischer Breite.

Klinisch relevante Interaktionen zwischen Substraten sind eher selten.

\section{CYP3A4: Das Iso-Enzym mit dem breitesten Substratspektrum}

CYP3A4-vermittelte AMI sind außerordentlich vielfältig, da dieses Iso-Enzym ein besonders breites Substratspektrum besitzt [2] ( $\triangleright$ Abb.4). Etwa $50 \%$ aller metabolisierten AM werden über CYP3A4 verstoffwechselt.
Relevant sind AMI insbesondere bei AM mit geringer therapeutischer Breite wie Antikoagulanzien. Sowohl Phenprocoumon als auch die NOAK Apixaban, Rivaroxaban und Edoxaban werden durch CYP3A4 abgebaut. So kann es beispielsweise bei Kombination von Phenprocoumon mit Verapamil, einem potenten CYP3A4Inhibitor, zu einem erhöhten Blutungsrisiko kommen. Bei Neuverordnung von CYP3A4-Induktoren oder -Inhibitoren sollte daher bei mit Phenprocoumon antikoagulierten Patienten die INR wenigstens einmal außerhalb der Routine bestimmt werden - am besten 3-5 Tage nach Therapiebeginn [18].

Aufgrund der fehlenden Möglichkeit des Monitorings der Gerinnungshemmung sind AMI bei NOAK besonders gefährlich. Rivaroxaban und Apixaban sind Substrate von CYP3A4 und P-gp. Die Kombination mit Pharmaka, die beide Eliminationswege hemmen, wie Azol-Antimykotika (z. B. Ketoconazol) oder HIV-Protease-Inhibitoren (z.B. Ritonavir), sollte vermieden werden ( $>$ Abb.5) [19]. Im klinischen Alltag wird jedoch auch viel zu häufig die kontraindizierte Kombination von Ticagrelor mit starken CYP3A4-Inhibitoren übersehen, dennTicagrelor wird hauptsächlich durch CYP3A4 zu seinem aktiven Metaboliten metabolisiert. Als Beispiel sei hier ein hospitalisierter Patient nach Stentimplantation bei akutem Koronarsyndrom genannt, der nun antibiotisch mit Clarithromycin behandelt wird.

Ferner bietet eine Kombination von CYP3A4-verstoffwechselten Statinen und einigen Makrolidantibiotika wie Clarithromycin und Erythromycin schwerwiegende Interaktionsmöglichkeiten. Hier kann es zu schweren Myopathien und Rhabdomyolysen kommen [20].

Cave

Viel zu oft wird in der Klinik die absolute Kontraindikation einer Kombination von Simvastatin und Clarithromycin oder Erythromycin übersehen.

Fluvastatin, Pravastatin und insbesondere Rosuvastatin (auch Pitavastatin) könnten Alternativen darstellen, da sie nicht exzessiv über CYP3A4 metabolisiert werden und weniger wahrscheinlich Rhabdomyolysen verursachen. Trotzdem müssen hier ebenfalls ein strenges Monitoring und eine Aufklärung der Patienten erfolgen, da auch hier AMI - obwohl nicht prospektiv untersucht beschrieben sind [21]. Hier sollte insbesondere auf klinische Symptome wie Myalgien oder Muskelschwäche geachtet werden und ggf. die Kreatinkinase-Aktivität bestimmt werden. Es ist aber vorzuziehen, die Kombination von Statinen mit Clarithyromycin/Erythromycin zu vermeiden durch Gabe eines anderen Antibiotikums, ggf. auch durch kurzzeitiges Pausieren des Statins. Letzteres muss in Abwägung des kardiovaskulären Risikos erfolgen. Grundsätzlich sind bei der Statin-Therapie 
auch andere Inhibitoren des Cytochrom-P450-Systems wie z. B. Verapamil und Diltiazem zu berücksichtigen.

Das in Zitrusfrüchten (besonders Grapefruit) enthaltene Naringin ist ein potenter Inhibitor von CYP3A4. Der Genuss eines Glases Grapefruitsaft führt zur irreversiblen Hemmung des gesamten intestinalen CYP3A4-Pools für 24 - 48 Stunden und erhöht damit die Bioverfügbarkeit von oral eingenommenen CYP3A4-Substraten. Der Anteil dieses sog. präsystemischen Metabolismus am Gesamtmetabolismus eines AM ist z. T. sehr hoch. Es kommt dann zu einem erheblichen Anstieg der Plasmakonzentration des AM bis um das 9-Fache (Simvastatin, Buspiron). Für Gerinnungshemmer wie Phenprocoumon ist die klinische Relevanz umstritten, allerdings sollten übermäßige Mengen an Grapefruitsaft bei antikoagulierten Patienten vermieden werden [2].

Bemerkenswert ist außerdem, dass einige hochpotente Opioide (Buprenorphin, Fentanyl, Oxycodon) ebenfalls CYP3A4-Substrate darstellen. Vorsicht ist hier insbesondere bei transdermalen Applikationsformen, die oft zur Therapie chronischer Schmerzen eingesetzt werden, geboten (s. „Fallbeispiel - CYP3A4-Inhibition“ [22]).

\section{FALLBEISPIEL \\ CYP3A4-Inhibition}

Eine 72-jährige Patientin mit Mammakarzinom und Knochenmetastasen erhält als Schmerztherapie Fentanyl-Pflaster. Während eines Krankenhausaufenthalts wird ihr zur Therapie einer Atemwegsinfektion Clarithromycin verabreicht. 2 Tage später entwickelt die Patientin plötzlich eine Ateminsuffizienz und wird bewusstlos. Nach sofortiger Gabe von Sauerstoff, Infusion von Naloxon und Absetzen des Fentanyls bessert sich der Zustand der Patienten im Laufe des darauffolgenden Tages [22].

CYP3A4-Induktoren sind insbesondere ältere Antiepileptika wie Carbamazepin und Phenytoin, aber auch das Tuberkulostatikum Rifampicin oder Hyperforin (Hauptwirkstoff in Johanniskrautextrakten).

\footnotetext{
Merke

Auf Hyperforin soll hier besonders hingewiesen werden, weil Johanniskrautextrakte von Patienten im Rahmen der Selbstmedikation oft vom Arzt unbemerkt eingenommen werden.
}

Insbesondere für Substanzen mit enger therapeutischer Breite kann der durch Induktion von CYP3A4 hervorgerufene schnellere Abbau schwerwiegende Folgen haben. Beispielhaft sei hier eine mögliche Transplan- tatabstoßung aufgrund eines Abfalls der Blutkonzentration von Ciclosprorin genannt.

Auch Ethinylestradiol als wesentlicher Bestandteil von oralen Kontrazeptiva wird durch CYP3A4 abgebaut. Bei Kombination mit Produkten mit Johanniskrautextrakten (Hyperforin) kann es zu vermehrtem Auftreten von Zwischenblutungen aufgrund des gesteigerten Abbaus von Ethinylestradiol kommen. Allerdings gibt es keine Evidenz dafür, dass die kontrazeptive Wirkung der Pille dadurch eingeschränkt ist [23].

\section{CYP2D6 vermittelt wichtige AMI der Psychopharmaka}

Selektive SSRI werden oft aufgrund des geringeren Potenzials für unspezifische AM-Wirkungen den trizyklischen Antidepressiva vorgezogen. Allerdings sind insbesondere Fluoxetin und Paroxetin potente Inhibitoren von CYP2D6, das immerhin den Abbau von $25 \%$ der metabolisierten AM vermittelt ( $\mathbf{A b b} \mathbf{3}$ u. $>\mathbf{A b b} \mathbf{4 b}$ ). Als Substrate gelten insbesondere Psychopharmaka wie trizyklische Antidepressiva, Antipsychotika und SSRI selbst. Hierauf sollte nicht nur bei Kombination dieser AM geachtet werden, sondern auch bei der Umstellung von Fluoxetin auf andere Psychopharmaka. Dabei sollte mit niedriger Dosis begonnen und die lange Halbwertszeit von Fluoxetin (4-6 Tage) berücksichtigt werden, welche durch die Hemmung des eigenen Abbaus bedingt ist.

Fluoxetin und Paroxetin hemmen auch den Abbau von Betarezeptorblockern und können so Bradykardie, AVBlockierungen und Blutdrucksenkung auslösen (s. „Fallbeispiel-CYP2D6-Inhibition“ [24]). Außerdem kann die Umwandlung von Codein und Tramadol in die aktiven Formen Morphin und O-Desmethyltramadol unterdrückt werden, wodurch die Gefahr des Therapieversagens besteht.

\section{FALLBEISPIEL \\ CYP2D6-Inhibition}

Ein 54 Jahre alter Patient erhält nach einer koronararteriellen Bypass-Operation täglich $100 \mathrm{mg}$ Metoprolol. Nach 4 Wochen entwickelt er eine beginnende Depression und der Hausarzt verordnet ihm 20 mg Fluoxetin pro Tag. Am übernächsten Tag klagt der Patient über Müdigkeit und Antriebsarmut. Im EKG wird eine ausgeprägte Bradykardie mit 36 Schlägen pro Minute festgestellt. Nach Absetzen von Fluoxetin kehrt die Herzfrequenz wieder auf Normalwerte zurück [24]. 


\section{CYP2C19: Aktivierung von Clopidogrel}

Das Iso-Enzym CYP2C19 spielt eine wesentliche Rolle bei der Aktivierung des Thrombozytenaggregationshemmers Clopidogrel ( $\mathbf{A b b . 4 c )}$ ). Zur Prävention von gastrointestinalen Blutungen werden Thrombozytenaggregationshemmer oft mit Protonenpumpeninhibitoren (PPI) kombiniert. Insbesondere Omeprazol und Lansoprazol stellen allerdings effektive Inhibitoren von CYP2C19 dar. Entsprechend konnte in Ex-vivo-Analysen und retrospektiven Beobachtungsstudien eine verminderte thrombozytenaggregationshemmende Wirkung von Clopidogrel und ein erhöhtes Risiko für kardiovaskuläre Ereignisse nachgewiesen werden. Im Gegensatz dazu konnten randomisierte, kontrollierte Studien keine Zunahme ischämischer Ereignisse oder Todesfälle erkennen. Vor dem Hintergrund dieser widersprüchlichen Daten schlagen Experten in einem Positionspapier der European Society of Cardiology vor, PPI mit einem geringeren CYP2C19-Interaktionspotenzial, z. B. Pantoprazol, zu bevorzugen.

\section{Merke}

Für die neueren Plättchen-Aggregationshemmer Prasugrel und Ticagrelor existieren keine Daten, die auf eine bedenkliche Interaktion mit PPI hinweisen [25].

\section{CYP2C9, CYP1A2}

AMI über CYP1A2 und CYP2C9 spielen klinisch eine eher untergeordnete Rolle. Hier sollen daher nur wenige Beispiele zur Veranschaulichung erwähnt werden.

Zu den Substraten von CYP1A2 zählt neben Theophyllin auch Koffein. Inhibiert wird CYP1A2 durch Ciprofloxacin und Norfloxacin. Polyzyklische aromatische Kohlenwasserstoffe, wie sie beispielsweise beim Rauchen ins Blut gelangen, induzieren CYP1A2. Dies führt bei Rauchern zu einer bis zu $50 \%$ reduzierten Halbwertszeit von Theophyllin.

Wichtige Substrate von CYP2C9 sind Warfarin und NSAR wie Ibuprofen, Diclofenac und Naproxen. An dieser Stelle soll auf die Interaktion des insbesondere im angloamerikanischen Raum gebräuchlichen Warfarins mit Amiodaron, einem CYP2C9-Inhibitor, hingewiesen werden.

\section{Vorgehen im Alltag}

Bei der Verschreibung neuer Medikamente müssen bereits bestehende Verordnungen auf mögliche AMI überprüft werden. Dabei sollen auch Nahrungsmittel, pflanzliche Präparate und eventuelle Selbstmedikation des Patienten erfragt und berücksichtigt werden.

Wichtige AMI haben wir in dieser Arbeit anhand klinisch relevanter Beispiele zusammengefasst. Darüber hinaus bieten Online-Hilfen oder entsprechende Softwarelösungen die Möglichkeit, Medikamentenlisten schnell auf Interaktionsmöglichkeiten zu prüfen bzw. vor diesen routinemäßig bei Verordnung neuer Medikamente zu warnen (s. „Praxis - Online Tools“).

\section{PRAXIS}

\section{Online Tools}

- Interaktionsdatenbanken:

- http://www.drugs.com/drug_interactions. php

- http://reference.medscape.com/drug-interactionchecker

- http://www.drug-interactions.com

- QT-Zeit verlängernde Medikamente, inkl. App:

- http://crediblemeds.org/

- Smartphone-Apps (für Apple iOS und Android):

- http://www.ifap.de/mobile-loesungen/

- http://www.medscape.com/public/medscapeapp

- http://www.epocrates.com/

\section{KERNAUSSAGEN}

- 1 - $5 \%$ aller Krankenhauseinweisungen sind auf Arzneimittelinteraktionen (AMI) zurückzuführen oder stehen mit diesen in Zusammenhang.

- Pharmakodynamische AMI können die Auftretenswahrscheinlichkeit sonst kaum beobachteter unerwünschter Arzneimittelwirkungen derart erhöhen, dass diese klinische Relevanz erhalten.

- Lediglich für die Kombination zweier AM mit „bekanntem Risiko“ für TdP-Arrhythmien konnte eine additive Wirkung auf die QT-ZeitVerlängerung nachgewiesen werden.

- Pharmakokinetische Interaktionen umfassen Wechselwirkungen, welche die Resorption, Verteilung, Metabolisierung und Elimination beeinflussen und die Konzentration am Wirkort verändern.

- P-Glykoprotein und Cytochrom-P450-Enzyme spielen eine wichtige Rolle bei pharmakokinetischen Interaktionen.

\section{Schlüsselwörter}

Arzneimittelinteraktionen, Cytochrom P450, Polypharmazie, Pharmakokinetik, Pharmakodynamik

\section{Erstveröffentlichung}

Dieser Beitrag wurde erstveröffentlicht in: Dtsch Med Wochenschr 2019; 144: 264-275. 


\section{Interessenkonflikt}

Die Arbeit der Autoren wird unterstützt durch die Deutsche Forschungsgemeinschaft (DFG VO 1568/3-1, IRTG1816 RP12 und SFB1002 TPA13 an Niels Voigt) und die Else-Kröner-Fresenius-Stiftung (EKFS 2016_A20 an Niels Voigt).

\section{Autorinnen/Autoren}

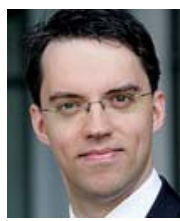

\section{Niels Voigt}

Prof. Dr. med., 2000-2007 Studium der Humanmedizin an der Technischen Universität Dresden. 2013 Facharzt für Pharmakologie und Toxikologie. 2015 Habilitation im Fach Pharmakologie und Toxikologie. Seit 2016

Professor für molekulare Pharmakologie an der Universitätsmedizin Göttingen. Schwerpunkte: Regulation von lonenkanälen und Veränderungen der Kalziumhomöostase am gesunden und kranken Herzen, insbesondere Herzrhythmusstörungen.

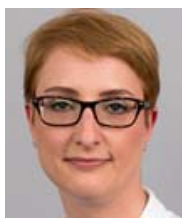

\section{Katharina Ort}

2003-2011 Studium der Humanmedizin an der Universitätsmedizin der Georg-August-Universität Göttingen. Seit 2011 Assistenzärztin in der Thorax-, Herz- und Gefäßchirurgie der Universitätsmedizin Göttingen und in der Ausbildung zur Fachärztin für Herzchirurgie. Schwerpunkte: Veränderungen der Kalziumhomöostase bei Herzrhythmusstörungen und Kardiomyopathien, herzchirurgische Intensivmedizin, v. a. postoperatives Delir und Sepsis/ SIRS.

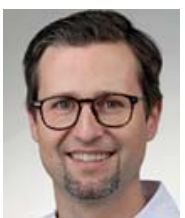

\section{Samuel Sossalla}

Prof. Dr. med., Leitender Oberarzt und Professor für experimentelle und interventionelle Kardiologie am Universitätsklinikum Regensburg. Experimentelle und klinische Schwerpunkte: Herzinsuffizienz, interventionelle Kardiologie, Behandlung von Rhythmusstörungen, pharmakologische Therapie von Herz-Kreislauf-Erkrankungen.

\section{Korrespondenzadresse}

Prof. Dr. med. Niels Voigt

Institut für Pharmakologie und Toxikologie Universitätsmedizin Göttingen, Georg-August-Universität Robert-Koch-Straße 40

37075 Göttingen

E-Mail: niels.voigt@med.uni-goettingen.de www.molecular-pharmacology.de

Wissenschaftlich verantwortlich gemäß Zertifizierungsbestimmungen

Wissenschaftlich verantwortlich gemäß Zertifizierungsbestimmungen für diesen Beitrag ist Prof Dr. med. Niels Vogt, Göttingen.
Literatur

[1] Dechanont S, Maphanta S, Butthum B et al. Hospital admissions/visits associated with drug-drug interactions: a systematic review and meta-analysis. Pharmacoepidemiol Drug Saf 2014; 23: 489-497

[2] Cascorbi I. Drug interactions - principles, examples and clinical consequences. Dtsch Arztebl Int 2012; 109: 546 555; quiz 556

[3] Kloner RA, Goldstein I, Kirby MG et al. Cardiovascular safety of phosphodiesterase type 5 inhibitors after nearly 2 decades on the market. Sex Med Rev 2018. doi:10.1016/j. sxmr.2018.03.008 [Epub ahead of print]

[4] Schwartz BG, Kloner RA. Drug interactions with phosphodiesterase- 5 inhibitors used for the treatment of erectile dysfunction or pulmonary hypertension. Circulation 2010; 122: $88-95$

[5] Pringle SD, MacEwen CJ. Severe bradycardia due to interaction of timolol eye drops and verapamil. Brit Med J 1987; 294: $155-156$

[6] de Abajo FJ. Effects of selective serotonin reuptake inhibitors on platelet function: mechanisms, clinical outcomes and implications for use in elderly patients. Drugs Aging 2011; 28: $345-367$

[7] Hackam DG, Mrkobrada M. Selective serotonin reuptake inhibitors and brain hemorrhage: a meta-analysis. Neurology 2012; 79: $1862-1865$

[8] Loke YK, Trivedi AN, Singh S. Meta-analysis: gastrointestinal bleeding due to interaction between selective serotonin uptake inhibitors and non-steroidal anti-inflammatory drugs. Aliment Pharmacol Ther 2008; 27: $31-40$

[9] Heijman J, Voigt N, Carlsson LG et al. Cardiac safety assays. Curr Opin Pharmacol 2014; 15: 16-21

[10] Drew B], Ackerman M], Funk M et al. Prevention of torsade de pointes in hospital settings: a scientific statement from the American Heart Association and the American College of Cardiology Foundation. Circulation 2010; 121: 1047 1060

[11] Meid AD, von Medem A, Heider D et al. Investigating the additive Interaction of QT-prolonging drugs in older people using claims data. Drug Saf 2017; 40: $133-144$

[12] Meid AD, Bighelli I, Machler S et al. Combinations of QTcprolonging drugs: towards disentangling pharmacokinetic and pharmacodynamic effects in their potentially additive nature. Ther Adv Psychopharmacol 2017; 7: 251-264

[13] Hohlfeld T, Saxena A, Schror K. High on treatment platelet reactivity against aspirin by non-steroidal anti-inflammatory drugs - pharmacological mechanisms and clinical relevance. Thromb Haemost 2013; 109: 825-833

[14] Heidbuchel H, Verhamme P, Alings M et al. Updated European Heart Rhythm Association Practical Guide on the use of nonvitamin $\mathrm{K}$ antagonist anticoagulants in patients with non-valvular atrial fibrillation. Europace 2015; 17: 1467 1507

[15] Benet LZ, Hoener BA. Changes in plasma protein binding have little clinical relevance. Clin Pharmacol Ther 2002; 71: $115-121$

[16] Shitara Y, Hirano M, Sato $\mathrm{H}$ et al. Gemfibrozil and its glucuronide inhibit the organic anion transporting polypeptide 2 (OATP2/OATP1B1:SLC21A6)-mediated hepatic uptake and CYP2C8-mediated metabolism of cerivastatin: analysis of the mechanism of the clinically relevant drug-drug interac- 
tion between cerivastatin and gemfibrozil. ] Pharmacol Exp Ther 2004; 311: $228-236$

[17] Haffner S, Thürmann PA. Which drug interactions the general practitioner should know. Med Klin 2004; 99: 137 -146; quiz 147

[18] Abbas S, Ihle P, Harder S et al. Risk of bleeding and antibiotic use in patients receiving continuous phenprocoumon therapy. A case-control study nested in a large insurance- and population-based German cohort. Thromb Haemost 2014; 111: $912-922$

[19] Dempfle CE. Pharmacology of the new oral anticoagulants. Herz 2012; 37: $362-368$

[20] Wagner ], Suessmair C, Pfister HW. Rhabdomyolysis caused by co-medication with simvastatin and clarithromycin. J Neurol 2009; 256: 1182-1183

[21] Li DQ, Kim R, McArthur E et al. Risk of adverse events among older adults following co-prescription of clarithromycin and statins not metabolized by cytochrome P450 3A4. CMAJ 2015; 187: $174-180$
[22] Cronnolly B, Pegrum H. Fentanyl-clarithromycin interaction. BMJ Case Rep 2012. doi:10.1136/bcr.02.2012.5936

[23] Hall SD, Wang Z, Huang SM et al. The interaction between St John's wort and an oral contraceptive. Clin Pharmacol Ther 2003; 74: 525-535

[24] Walley T, Pirmohamed M, Proudlove $C$ et al. Interaction of metoprolol and fluoxetine. Lancet 1993; 341: $967-968$

[25] Agewall S, Cattaneo M, Collet JP et al. Expert position paper on the use of proton pump inhibitors in patients with cardiovascular disease and antithrombotic therapy. Eur Heart J 2013; 34: $1708-1713,1713 a-1713 b$

Bibliografie

DOI https://doi.org/10.1055/a-0863-6242

Pneumologie 2019; 73: 306-318

(c) Georg Thieme Verlag KG Stuttgart · New York ISSN 0934-8387 


\section{Punkte sammeln auf CME.thieme.de}

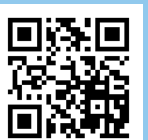

Diese Fortbildungseinheit ist in der Regel 12 Monate online für die Teilnahme verfügbar.

Den genauen Einsendeschluss finden Sie unter https://eref.thieme.de/CXCQRU8.

Sollten Sie Fragen zur Online-Teilnahme haben, finden Sie unter https://cme.thieme.de/hilfe eine ausführliche Anleitung. Wir wünschen viel Erfolg beim Beantworten der Fragen!

Unter https://eref.thieme.de/CXCQRU8 oder über den QR-Code kommen Sie direkt zur Startseite des Wissenstests.

VNR 2760512019156640755

\section{Frage 1}

Wie viel Prozent der jährlichen Krankenhauseinweisungen stehen ganz oder teilweise im Zusammenhang mit Arzneimittelinteraktionen (AMI)?
A $\quad 10-50 \%$
B $1-5 \%$
C $0,1-0,5 \%$
D $\quad 0,01-0,05 \%$
E $1,5-15 \%$

\section{Frage 2}

Welche Aussage zu Selektiven Serotonin-RückaufnahmeInhibitoren (SSRI) ist falsch?
A SSRI hemmen die Wiederaufnahme von Serotonin in serotoninerge Neuronen.
B SSRI hemmen die Aufnahme von Serotonin in Thrombo- zyten.
C SSRI haben keinen Einfluss auf das Blutungsrisiko unter Therapie mit oralen Antikoagulanzien.
D Fluoxetin steigert die Halbwertszeit von Haloperidol.
E Paroxetin hemmt den eigenen Abbau.

\section{Frage 3}

Welches Medikament weist nach der AZCERT-Klassifikation ein „bekanntes Risiko“ für das Auftreten von Torsade-de-PointesTachykardien (TdP) auf?
A Tramadol
B Omeprazol
C Clozapin
D Citalopram
E Loperamid

\section{Frage 4}

Welcher der folgenden Wirkstoffe kann das Auftreten einer Atemdepression unter Therapie mit Fentanyl begünstigen?
A Omeprazol
B Clarithromycin
C Amoxicillin
D Metronidazol
E Rifampicin

\section{Frage 5}

Welche Kombination von CYP-Isoenzym und dazugehörigem Inhibitor ist falsch?
A CYP2C19: Omeprazol
B CYP2D6: Fluoxetin
C CYP3A4: Rifampicin
D CYP2C9: Amiodaron
E CYP1A2: Ciprofloxacin

\section{Frage 6}

Bei welchem Medikament führt die Hemmung eines entsprechenden CYP-Isoenzyms zu einer Wirkverstärkung?
A Codein
B Tramadol
C Clopidogrel
D Prasugrel
E Fentanyl 


\section{Frage 7}

Welche Aussage zu den neuen oralen Antikoagulanzien (NOAK) trifft zu?

A NOAKs sind bei Patienten mit Niereninsuffizienz besonders geeignet.

B Dabigatran wird über CYP3A4 metabolisiert.

C Klinisch relevante Blutungen treten unter den NOAKs häufiger auf.

D Unter Therapie mit Verapamil ist mit einer erhöhten Bioverfügbarkeit von Dabigatran zu rechnen.

E Dabigatranetexilat wird sehr gut aus dem Darmlumen resorbiert

\section{Frage 8}

Auch Nahrungs- und Genussmittel sowie frei verkäufliche Präparate aus Drogerien und Apotheken bergen die Gefahr von Arzneimittelinteraktionen (AMI). Welche der folgenden Zuordnungen von Substanz und relevantem Mechanismus der Interaktion ist am ehesten zutreffend?
A Milch - Hemmung von p-Glykoprotein
B Johanniskraut - CYP3A4-Induktor
C Grapefruit-CYP3A4-Induktor
D Koffein - Bildung schwerlöslicher Chelate
E Tabakrauch - gesteigerte Expression von verschiedenen Transportproteinen der ABC-Familie

\section{Frage 9}

Welche Aussage zur Einnahme von nichtsteroidalen Antirheumatika (NSAR) bei Patienten, die bereits mit Acetylsalicylsäure (ASS) behandelt werden, ist richtig?

A Die Einnahme des NSAR sollte mindestens $30 \mathrm{~min}$ vor Einnahme von ASS erfolgen.

B Ibuprofen verstärkt die thrombozytenaggregationshemmende Wirkung von ASS.

C Paracetamol reduziert die thrombozytenaggregationshemmende Wirkung von ASS.

D NSAR hemmen kompetitiv den Abbau von ASS.

E Einmalige Einnahme von NSAR unter ASS-Therapie ist unbedenklich.

\section{Frage 10}

Für welches Statin besteht eine absolute Kontraindikation hinsichtlich der Kombination mit Clarithromycin oder Erythromycin?
A Pitavastatin
B Fluvastatin
C Pravastatin
D Rosuvastatin
E Simvastatin 